\title{
Managing Bipolar Disorder in Time of COVID-19 Pandemic
}

\author{
Rabie KARROURI ${ }^{1}$, Zakaria HAMMANI ${ }^{2}$, Yassine OTHEMAN ${ }^{3 *}$ \\ ${ }^{1}$ Assistant Professor of Psychiatry. Psychiatry department. Moulay Ismail Military Hospital. Faculty of medicine and pharmacy. Sidi Mohamed \\ Ben Abdellah University. Fez, Morocco. \\ ${ }^{2}$ Psychiatrist. Psychiatry department. Moulay Ismail Military Hospital. \\ ${ }^{3}$ Associate Professor of Psychiatry. Head of Psychiatry department. Moulay Ismail Military Hospital. Faculty of medicine and pharmacy. Sidi \\ Mohamed Ben Abdellah University. Fez, Morocco.
}

\begin{abstract}
People with bipolar disorder are at high risk of serious complications during the COVID-19 pandemic. Limited access to care, lifestyle, health condition and other clinical characteristics increase the risk of contracting the new coronavirus disease, with more risk of developing severe forms, as well as that of bipolar relapse. Here, we will describe some problematic situations that may be encountered by bipolar patients during this pandemic period, and the measures that can be taken to reduce the impact of such problems on bipolar disorder course. Maintaining a daily schedule with relaxing and pleasing activities, getting enough sleep, keeping contact with mental health professionals and continuing prescribed medication, are important measures to prevent relapse. Family and friends, have also a key role in accompanying and supporting patients in these crisis time.
\end{abstract}

\section{KEYWORDS}

COVID-19, Bipolar disorders, Pandemic, Relapse, Mania, Depression.

\section{Correspondins Author Information}

\section{Yassine Otheman}

Associate Professor of Psychiatry. Head of Psychiatry department. Moulay Ismail Military Hospital. Faculty of medicine and pharmacy. Sidi Mohamed Ben Abdellah University. Fez, Morocco.

Received: April 30, 2020; Accepted: May 25, 2020; Published: June 01, 2020

Copyright: (C) 2020 ASRJS. This is an openaccess article distributed under the terms of the Creative Commons Attribution 4.0 International license.

Citation: Rabie KARROURI, Zakaria HAMMANI, Yassine OTHEMAN. Managing Bipolar Disorder in Time of COVID-19 Pandemic. Med Clin Res Open Access, 2020;1(1):1-4.

\section{Introduction}

During the COVID-19 pandemic, people with severe mental illness are at high risk of serious complications. On one hand, limited access to care and confinement conditions can precipitate relapses and worsening of psychiatric illness. On the other hand, their lifestyles increase the risk of contracting the new coronavirus disease, with more risk of developing severe forms, because of their mental status and underlying health conditions.
One of the conditions, that expose the most to such risks, is bipolar mood disorder, which is a common mental disorder affecting 2 to $3 \%$ of the general population (1). This disorder, by its clinical, etiopathological and socio-familial implications, can pose serious problems, during this pandemic, to the patient and those around him, and put mental health professionals in front of the challenge of preventing decompensations and treating relapses in time. In this article, we will describe some problematic situations that may be encountered by bipolar patients during this pandemic period, 
and the measures that can be taken to reduce the impact of such problems on bipolar disorder course.

\section{Bipolar disorders}

Bipolar mood disorders are a set of chronic psychiatric disorders characterized by the onset of manic or hypomanic episodes, whether or not associated with depressive episodes. These pathological variations in mood are often accompanied by variations in energy, sleep and behavior. In its most severe forms, we can observe risky behavior, psychotic symptoms (delusion, hallucination, etc.), aggressiveness or suicidal behavior. The causes of this disease are not exactly known, but we do know that genetic, neurobiochemical and environmental factors are involved in the etiopathogenesis of the disorder. The treatment is based on mood stabilizer medications, associated, if necessary, with other types of psychotropic medication according to the clinical symptoms; while psychotherapy and a healthy lifestyle with, above all, a regular rhythm of life, are very useful for the prevention of relapses (2).

\section{Problems facing bipolar patients during COVID-19 crisis Stress}

Patients with bipolar disorders are strongly reactive to stress (3). During this covid pandemic, several factors combined to lead to a situation of continuous stress: the fear of being infected or that of a loved one, the flow of information concerning the pandemic and resulting deaths, loss of job or income linked to the economic crisis, the limitation of access to outside entertainment facilities and the absence of relatives... All these factors added to other difficulties that we will address here, have created a deleterious stressful situation for these patients.

\section{Daily life changes}

The confinement and the changes of usual daily activities, leads to lost of the structuring of daily life, witch is one of the most challenging aspects of this pandemic for bipolar patients. These changes are often responsible for disturbing daily life rhythms, with an alteration in the quality of sleep. Patients with bipolar disorder are more sensitive to these changes, especially those of sleep. Loss of sleep is a trigger and one of the first signs of relapse, and even one night of missed sleep may lead to manic symptoms (4).

\section{Difficulty to access support and care}

Restricted travel during this pandemic period has resulted in reduced opportunities for access to psychological and medical support and care. Access to care has become less easy, with longer appointment times. Patients are sometimes forced to consult professionals other than those they used to see, and who are reassuring figures for them.

In several healthcare structures, notably those of general hospitals managing covid patients, the supply of psychiatric care has been reduced to allow teams to focus on managing the pandemic. Furthermore, access to hospital has become less secure with the risk of being contaminated. Among other things, non-essential activities like psychoeducation, individual therapy session for stabilized patients and group therapy sessions have been postponed.
Outpatients visits are often limited to the most urgent cases, and hospitalizations to the most serious cases. This drop in the supply of care may weaken patients with chronic relapsing pathologies like bipolar disorders.

In addition, social contacts have also been reduced due to social distancing. The role of loved ones in supporting bipolar patients is important, not to mention their role in detecting the first symptoms of relapses (5). This is all the more important since bipolar patients, particularly in the manic or hypomanic phase, are not often aware of their disorders, given the lack of insight that characterizes these patients during these episodes (6). The lack of socio-psychological support and medical assistance makes bipolar patients even more fragile, especially when they need therapeutic adjustment or therapy sessions.

\section{Specific problems related to bipolar disorders}

During an episode of manic excitement, patients may exhibit risky behaviors with serious consequences during this pandemic period. Disinhibition and ideas of greatness and superpower, or even delusions that can characterize these episodes, can lead to many behavioral disorders. Thus, these patients can break the rules of confinement, challenge authorities, feel infallible in front of the disease, or even think they have a truth that goes against what is accepted in the scientific sphere, including the contagious nature of the disease or unusual treatments that can be dangerous for COVID 19 patients.

On the other hand, during episodes of depression, bipolar patients may not seek help if they contract the disease, especially when the episode is accompanied by thoughts of incurability, hopelessness, or suicidal ideation. Social isolation can aggravate this situation while patients are most in need of support and presence of loved ones.

\section{Addictive comorbidity}

Bipolar disorder is highly associated with addictive comorbidity $(7,8)$. During these times of confinement and stress, patients will tend to use recreational drugs to seek relief from their anxiety and emotional pain. This often progresses to regular consumption, which is often problematic for these patients, and causes more frequent relapses, more severe forms and a less favorable response to treatment (9). Substance use is another serious problem to manage during this period.

\section{Problems related to news exposure}

During this pandemic period, erroneous information of all kinds circulates easily in the media, in particular social media and sites defending conspiracy theories. This seriously harms public health policies and affects the most vulnerable populations, including those suffering from psychiatric disorders, especially of psychotic or bipolar type, who are more inclined to adhere to conspiracy ideas and para-scientific explanations. These patients are therefore more at risk for contracting the disease without using official healthcare system. They are also at risk of stopping psychiatric treatment that may be wrongly accused of reducing their immunity 
and increasing the risk of being infected.

\section{Bipolar disorders and COVID-19 comorbidity}

One of the most problematic situations that can arise during this period is the onset of COVID-19 in a not stabilized bipolar patient. It's common for patients with such mental illnesses to refuse medical care, even if they have symptoms, and when it comes to COVID-19, this can be a matter of life or death. Moreover, health condition in bipolar patient are generally less optimal than in other people. Rates of smoking are higher, diabetes, hypertension, heart disease, poor cholesterol and other somatic conditions are more frequent in bipolar patients, increasing their risk to experience more likely COVID-19 complications (10-12). Knowing that pneumonia and influenza are some of the leading causes of mortality in people with mental illness (13), this pandemic crisis represent a high-risk situation for bipolar patient.

\section{How to manage these problems?}

Respect daily life Rhythms

Bipolar patients do better with routinely lifestyle over time (14). Chronobiologic disturbance is one of the most incriminated factors in mood disorders and in particular bipolar disorders $(15,16)$. The best way to handle these times of confinement is to divide daytime into different segments of one to two hours, with the same kind of activities at the same time each day. Thus, several activities can be recommended: reading, exercising, watching programs, talking with friends and relatives online, cooking, gardening, drawing, meditating, playing video games etc. The most important is to create a program that helps structure the day and the week, by adopting the same schedules for meals and sleep as those which were before the pandemic.

\section{Maintain a healthy lifestyle}

This requires good sleep hygiene, regular physical activity and a balanced diet. Healthy sleep habits are very important for bipolar patients as are medications (17). In fact, sleep is a good clinical marker of the disorder evolution (18). Maintaining an active exercise routine, even at home, is very important in these times. Regular exercise is one of the most positive action to reduce stress consequences on body and spirit. Patients can choose online videos of exercise sessions that suit them and include them in the day's program. Regarding nutrition, balanced meals with, above all, the avoidance of any stimulant (coffee, tea, etc.) are generally recommended for these patients, and even more during this period when immunity must be maintained at its best.

\section{Avoid substance use}

Either to manage stress and anxiety or to feel good and in better shape, substance use may look like an easy solution for bipolar patients who want to overcome their symptoms. In addition to respecting daily rhythms of life and a healthy lifestyle in general, some stress management and anxiety reduction techniques can be used to avoid the use of substances. These techniques can be learned with mental health professionals, but also through exercises available online such as cardiac coherence or mindfulness meditation. Without forgetting to limit exposure to the continuous Vol 1 Iss 1 flow of information related to the pandemic and its consequences, without exceeding 10 to 15 minutes per day of exposure to news.

\section{Maintain adequate social support}

In times of extended social distancing, most of us engage in online social interaction, which is healthy for us emotionally. Bipolar patient may find it difficult to look actively for social connection, especially while depressed. This may be more important for older individuals who are not used to social media. Suffering people are more resilient to negative feelings when they receive support from meaningful persons (19). That's why it is very important to identify a person you know well and whom you can talk with regularly. Families and friends of bipolar patients should be more present and give support to their ill relatives during the COVID-19 pandemic.

\section{Adapt healthcare offer}

Psychiatrists will have to rapidly adapt to the situation and to patient's needs. One of the most helpful techniques in this pandemic crisis is teleconsultation (20). For most patients, telehealth appointment is a good way to support patients during this period, but for a minority, whose state of health requires it, an in person meeting should be always possible, and short-term hospitalization may be necessary when there is a risk of suicide or aggression, or when the patient refuses treatment, taking the necessary precautions to reduce the risk of contamination.

The patient and his family must remain in contact with the attending physician or the psychiatric department where the patient is followed up, to avoid any therapeutic deviation, and ensure an adequate supply of medication for a sufficient period ( 2 or 3 months), ensuring that pharmacist will agree to deliver distance prescription treatment.

\section{Adapt treatment to COVID-19 patients}

Patients with bipolar disorders, who suffer from COVID-19, may require a therapeutic adjustment to avoid certain drug interactions between psychotropic drugs and those used in the COVID-19 therapeutic protocol. Thus, the use of certain antipsychotics, used as mood stabilizers, or certain antidepressants, may increase the risk of heart rhythm disorders, especially torsades de pointes, especially with the combination of hydroxychloroquine / azithromycin (22). This involves a prior cardiac assessment and daily monitoring of the ECG, but also the switch to psychotropic molecules, with less cardiotropic effects, when needed. In addition, the use of benzodiazepines and hypnotics must be conditioned by the absence of risk of respiratory distress and liver failure, which contraindicates these molecules. In general, the concomitant use of psychotropic drugs and different medications that may be used in the management of patients with COVID-19, implies closer monitoring of different physiological functions, in particular cardiac, hepatic, renal, respiratory functions and blood count.

\section{Conclusion}

Bipolar patients decompensate more likely during times of stress and changes in life rhythms, like the one we are currently living. Furthermore, many key risk factors for serious COVID-19 Pages 3 of 4 
complications are also common in these patients. Maintaining routine and a daily schedule with relaxing and pleasing activities, getting enough sleep, keeping contact with mental health professionals and continuing prescribed medication, are important measures to prevent relapse. Family and friends, have a key role in accompanying and supporting patients, and must themselves preserve their well-being to be useful and comforting to patients.

\section{References}

1. American Psychiatric Association, DSM-5 Task Force. Diagnostic and Statistical Manual of Mental Disorders: DSM5 (5th ed.) American Psychiatric Publishing, Inc. 2013.

2. Dean OM, Gliddon E, Van Rheenen TE. An update on adjunctive treatment options for bipolar disorder. Bipolar Disord, 2018;20(2):87-96.

3. Muhtadie L, Johnson SL. Threat sensitivity in bipolar disorder. J Abnorm Psychol, 2015;124(1):93-101.

4. Lewis KS, Gordon-Smith K, Forty L. Sleep loss as a trigger of mood episodes in bipolar disorder: individual differences based on diagnostic subtype and gender. Br J Psychiatry, 2017;211(3):169-174.

5. Miklowitz DJ, Chung B. Family-Focused Therapy for Bipolar Disorder: Reflections on 30 Years of Research. Fam Process, 2016;55(3):483-499.

6. Otheman Y. Comparative study of insight and factors influencing it in schizophrenia and bipolar disorder in a population of Moroccan hospitalized patients. Ann Med Psychol (Paris), 2013.

7. Stokes PRA, Kalk NJ, Young AH. Bipolar disorder and addictions: the elephant in the room. Br J Psychiatry, 2017;211(3):132-134.

8. Varo C, Murru A, Salagre E. Behavioral addictions in bipolar disorders: A systematic review. Eur Neuropsychopharmacol, 2019;29(1):76-97.

9. Gold AK, Otto MW, Deckersbach T, Sylvia LG, Nierenberg
AA, Kinrys G. Substance use comorbidity in bipolar disorder: A qualitative review of treatment strategies and outcomes. Am J Addict, 2018;27(3):188-201.

10. Gonzalez-Pinto A, Gutierrez M, Ezcurra J. Tobacco smoking and bipolar disorder. J Clin Psychiatry. 1998;59(5):225-228.

11. Crump C, Sundquist K, Winkleby MA, Sundquist J. Comorbidities and mortality in bipolar disorder: a Swedish national cohort study. JAMA Psychiatry, 2013;70(9):931-939.

12. Sayuri Yamagata A, Brietzke E, Rosenblat JD, Kakar R, McIntyre RS. Medical comorbidity in bipolar disorder: The link with metabolic-inflammatory systems. J Affect Disord, 2017;211:99-106.

13. Dickerson F, Origoni A, Schroeder J, et al. Mortality in schizophrenia and bipolar disorder: Clinical and serological predictors. Schizophr Res, 2016;170(1):177-183.

14. Miklowitz DJ. A review of evidence-based psychosocial interventions for bipolar disorder. J Clin Psychiatry, 2006;67 Suppl 11:28-33.

15. Sigitova E, Fišar Z, Hroudová J, Cikánková T, Raboch J. Biological hypotheses and biomarkers of bipolar disorder. Psychiatry Clin Neurosci, 2017;71(2):77-103.

16. Dallaspezia S, Benedetti F. Chronobiology of bipolar disorder: therapeutic implication. Curr Psychiatry Rep, 2015;17(8):606.

17. Takaesu Y. Circadian rhythm in bipolar disorder: A review of the literature. Psychiatry Clin Neurosci, 2018;72(9):673-682.

18. Gold AK, Sylvia LG. The role of sleep in bipolar disorder. Nat Sci Sleep, 2016;8:207-214.

19. Ariapooran S, Heidari S, Asgari M, Ashtarian H, Khezeli M. Individualism-Collectivism, Social Support, Resilience and Suicidal Ideation among Women with the Experience of the Death of a Young Person. Int J Community Based Nurs Midwifery, 2018;6(3):250-259.

20. Wright JH, Caudill R. Remote Treatment Delivery in Response to the COVID-19 Pandemic. Psychother Psychosom, 2020;89(3):130-132. 